\section{Error-Constrained Filtering for a Class of Nonlinear Time-Varying Delay Systems With Non-Gaussian Noises}

\author{
Guoliang Wei, Zidong Wang, and Bo Shen
}

\begin{abstract}
In this technical note, the quadratic error-constrained filtering problem is formulated and investigated for discrete time-varying nonlinear systems with state delays and non-Gaussian noises. Both the Lipschitz-like and ellipsoid-bounded nonlinearities are considered. The non-Gaussian noises are assumed to be unknown, bounded, and confined to specified ellipsoidal sets. The aim of the addressed filtering problem is to develop a recursive algorithm based on the semi-definite programme method such that, for the admissible time-delays, nonlinear parameters and external bounded noise disturbances, the quadratic estimation error is not more than a certain optimized upper bound at every time step $k$. The filter parameters are characterized in terms of the solution to a convex optimization problem that can be easily solved by using the semi-definite programme method. A simulation example is exploited to illustrate the effectiveness of the proposed design procedures.
\end{abstract}

Index Terms-Non-Gaussian noises, nonlinear filtering, quadratic error constraints, semi-definite programme method, time delays, time-varying systems.

\section{INTRODUCTION}

Filtering problem is a widely studied research topic in the areas of control and signal processing. The main objective of the filtering problems is to estimate the unavailable state information or remove all kinds of noise signals from the noisy measurement information. During the past decades, various filtering problems have been extensively investigated and a large amount of results have been available, see, e.g., [6], [17], [21], [24], [29], [33], [34]. According to the types of noise signals and the performance criteria, several important filtering approaches have been developed in the literature including the Kalman filtering [12], [14], [15], [31], [33], $H_{\infty}$ filtering [4], [5], [7], [21], [29], [30], [32], optimal filtering [1]-[3] and variance-constrained filtering [11], [24], [35].

As is well known, time delays are often encountered in many practical systems, such as engineering system, network system, nuclear reactor, biological system, chemical system and electrical networks system. Therefore, the past few decades have witnessed significant progress on filtering and control for linear/nonlinear systems with various types of delays, and a great deal of literature has appeared on the general topic of time-delay systems, see, e.g. [5], [7], [12], [17], [27]. In particular, the filtering problem has been considered for the discrete time-varying systems with time-delays for its popular applications in digital signal processing, and some results have recently been published, see, e.g., [12].

Manuscript received May 19, 2009; revised February 10, 2010; accepted August 26, 2010. Date of publication September 07, 2010; date of current version December 02, 2010. This work was supported in part by the Leverhulme Trust of the U.K., the Engineering and Physical Sciences Research Council (EPSRC) of the U.K. under Grant GR/S27658/01, the Royal Society of the U.K., the National Natural Science Foundation of China under Grant 61028008 and Grant 61074016, the Shanghai Natural Science Foundation of China under Grant 10ZR1421200, and the Alexander von Humboldt Foundation of Germany. Recommended by Associate Editor E. Fabre.

G. Wei and B. Shen are with the School of Information Science and Technology, Donghua University, Shanghai 200051, China.

Z. Wang is with the Department of Information Systems and Computing, Brunel University, Uxbridge, Middlesex, UB8 3PH, U.K. (e-mail: zidong.wang@brunel.ac.uk).

Color versions of one or more of the figures in this technical note are available online at http://ieeexplore.ieee.org.

Digital Object Identifier 10.1109/TAC.2010.2073311
Non-Gaussian noises and nonlinearities exist universally in nature. First, the non-Gaussian noises are a kind of more general signals than the widely studied Gaussian noises [9], [10], [18]-[20], [26], [35]. Among others, the bounded noises are an important type of non-Gaussian noises that have stirred considerable research attention. In particular, the filtering problems have been extensively considered for systems with bounded noises, see, e.g., [8], [13], [23]. The nonlinear filtering problems, on the other hand, have been attracting constant research interests from many researchers in the past few decades, see [4], [15], [22], [27], [28] for some examples. Unfortunately, when the nonlinearities and non-Gaussian noises come together for time-varying systems with time-delays, the filtering problem has become quite involved due primarily to the degree of mathematical complexity where existing filtering techniques cannot be simply applied, despite its significance in engineering applications. For example, the Kalman filtering and variance-constrained filtering methods are based on the statistical assumptions on Gaussian-type noises, hence are no longer effective for systems with non-Gaussian noise disturbances. The $H_{\infty}$ filter has proven to be less sensitive to non-Gaussian noise. However, the traditional $H_{\infty}$ filtering approach does not take into account the estimation error bound that is an important performance index, and therefore $H_{\infty}$ filtering may lead to unnecessarily large conservatism in certain situations. It is, therefore, the aim of this technical note to develop a novel approach to handling the recursive filtering problems for nonlinear time-delay time-varying systems with bounded noises by a numerically appealing algorithm.

In this technical note, we introduce the quadratic error-constrained filtering problem for discrete time-varying nonlinear systems with state delays and non-Gaussian noises, and aim to develop an effective recursive filtering algorithm. The main contributions of this technical note can be summarized as follows: 1) both the Lipschitz-like and ellipsoidbounded nonlinearities are investigated for the addressed time-varying delay systems; 2) the quadratic estimation error is enforced on the recursive process at every time step; and 3) a semi-definite programme method is developed to deal with the recursive filtering problem for online applications. The filter parameters are characterized in terms of the solution to a convex optimization problem that can be easily solved by using the semi-definite programme method. A simulation example is exploited to illustrate the effectiveness of the proposed design procedures.

Notation: In this technical note, $\mathbb{R}^{n}, \mathbb{R}^{n \times m}, \mathbb{Z}^{+}$denote, respectively, the $n$-dimensional Euclidean space, the set of all $n \times m$ real matrices, the set of all positive integers. $|\cdot|$ refers to the Euclidean norm in $\mathbb{R}^{n} . I$ denotes the identity matrix of compatible dimension. The notation $X \geq Y$ (respectively, $X>Y$ ), where $X$ and $Y$ are symmetric matrices, means that $X-Y$ is positive semi-definite (respectively, positive definite). For a matrix $M, M^{T}$ and $M^{-1}$ represent its transpose and inverse, respectively. If $M$ is a square matrix, $\operatorname{trace}(M)$ denotes the trace of $M$. The shorthand $\operatorname{diag}\left\{M_{1}, M_{2}, \ldots, M_{n}\right\}$ denotes a block diagonal matrix with diagonal blocks being the matrices $M_{1}, M_{2}, \ldots, M_{n}$. Matrices, if they are not explicitly stated, are assumed to have compatible dimensions.

\section{PROBLEM Formulation}

Consider the following discrete time-varying nonlinear systems with time-delays and bounded noises:

$$
\begin{aligned}
x(k+1) & =f(x(k))+g(x(k-\tau))+D(k) \omega(k) \\
y(k) & =C(k) x(k)+E(k) \omega(k) \\
x(k) & =\phi, \quad \forall k=-\tau, \cdots, 0
\end{aligned}
$$


where $x(k) \in \mathbb{R}^{n}$ is the state, $y(k) \in \mathbb{R}^{q}$ is the measurement output, $\tau \in \mathbb{Z}^{+}$is a constant delay. $f(\cdot)$ and $g(\cdot)$ are nonlinear functions with $f(0)=0$ and $g(0)=0$. For a given time step $k, C(k), D(k)$ and $E(k)$ are constant matrices with appropriate dimensions. $\omega(k)$ is the disturbance noise, which is unknown, bounded and confined to the following specified ellipsoidal sets:

$$
\Psi_{S}(\omega(k))=\left\{\omega(k): \omega^{T}(k) S^{-1}(k) \omega(k) \leq 1\right\}
$$

where $S(k)$ is a known positive-definite matrix sequence with compatible dimensions, which denotes the "shape" of the ellipsoids with 0 being the center of the ellipsoids. The initial state vector $\phi$ satisfies the following quadratic error constraints:

$$
e_{0} e_{0}^{T}=\left(\phi-\phi_{f}\right)\left(\phi-\phi_{f}\right)^{T} \leq \Xi_{0}
$$

where $\phi_{f}$ is the given estimate of $\phi, e_{0}$ is the initial error between $\phi$ and $\phi_{f}$, and $\Xi_{0}>0$ is the given initial upper bound on the quadratic error $e_{0} e_{0}^{T}$.

Remark 1: In many practical systems, the disturbance noises are known to be non-Gaussian due to man-made electromagnetic interference and other natural sources, see, e.g., [9], [10], [26], [35]. Most existing filtering approaches, however, are based on the assumption that the noises are Gaussian [11], [24], [31]. Therefore, most conventional filtering methods are no longer applicable to the filtering problems subject to non-Gaussian noises and there is an urgent need to develop new filtering algorithms. In (3), the noises $\omega(k)$ are assumed to be unknown, bounded, deterministic but reside within in ellipsoidal sets.

In this technical note, we consider two kinds of descriptions for the nonlinear functions $f(\cdot)$ and $g(\cdot)$. In both cases, the quadratic errorconstrained filtering problem for (1), (2) will be investigated. For presentation convenience, we denote

$$
\begin{aligned}
D_{f}(x(k), \sigma(k)):= & f(x(k)+\sigma(k))-f(x(k)) \\
& -A(k) \sigma(k) \\
D_{g}(x(k), \sigma(k)):= & g(x(k)+\sigma(k))-g(x(k)) \\
& -B(k) \sigma(k) .
\end{aligned}
$$

Case 1: The nonlinear functions $f(x(k))$ and $g(x(k))$ satisfy the following Lipschitz-like conditions [22], [25]:

$$
\begin{aligned}
& \left|D_{f}(x(k), \sigma(k))\right| \leq a(k)|\sigma(k)|, \\
& \left|D_{g}(x(k), \sigma(k))\right| \leq b(k)|\sigma(k)|
\end{aligned}
$$

where $A(k)$ and $B(k)$ in (5), (6) are known matrices, and $a(k)$ and $b(k)$ are known positive scalars at every time step $k$.

Remark 2: The nonlinearity description in (7) quantifies the "distance" nature between the nonlinear systems (1), (2) and the corresponding linear systems with matrices $A(k), B(k)$. Note that the filtering problems have already been discussed for nonlinear systems with such a description, see [22], [25].

Case 2: The nonlinear functions $f(x(k))$ and $g(x(k))$ are constrained within the following ellipsoidal sets:

$$
\begin{aligned}
\Psi(\mathfrak{A}(k), A(k))=\{ & f(x(k)) \mid D_{f}^{T}(x(k), \sigma(k)) \mathfrak{A}^{-1}(k) \\
& \left.\times D_{f}(x(k), \sigma(k)) \leq 1\right\} \\
\Psi(\mathfrak{B}(k), B(k))=\{ & g(x(k)) \mid D_{g}^{T}(x(k), \sigma(k)) \mathfrak{B}^{-1}(k) \\
& \left.\times D_{g}(x(k), \sigma(k)) \leq 1\right\}
\end{aligned}
$$

where $A(k), B(k), \mathfrak{A}(k)>0$ and $\mathfrak{B}(k)>0$ are known matrices. Note that $A(k) \sigma(k)$ and $B(k) \sigma(k)$ can be understood as the centers of ellipsoids, and $\mathfrak{A}(k)$ and $\mathfrak{B}(k)$ are therefore the corresponding "shape" matrices.
Remark 3: The description in (8) is referred to as the confidence ellipsoid description. The so-called confidence ellipsoid has been extensively utilized to account for certain noises and filtering performance index, see e.g. [8], [16], [35]. Note that the distance between the nonlinear systems and the "nominal" linear models could be adjusted according to engineering requirements by changing the ellipsoidal "center" vectors and "shape" matrices. To the best of our knowledge, such a description represents the first of few attempts that uses confidence ellipsoids to define the nonlinear systems in a filtering problem.

In this technical note, we aim to construct the following time-varying filter for (1), (2):

$$
\begin{aligned}
x_{f}(k+1)=f\left(x_{f}(k)\right)+g\left(x_{f}(k-\tau)\right) & \\
& +L(k)\left(y(k)-C(k) x_{f}(k)\right)
\end{aligned}
$$

where $L(k)$ are the filter parameters to be designed.

The objective of this technical note is to design an optimal nonlinear filter (9) for the discrete time-varying nonlinear systems (1), (2) in the sense that the sequence of quadratic error constraint matrices $\Xi(k)$ is minimized. The detailed problem is formulated as follows: find the sequence $L(k)$ of the filter parameters as well as a sequence of positive-definite matrices $\Xi(k+1)$ to solve the optimization problem:

$$
\begin{aligned}
& \min _{\Xi(k+1), L(k)} \operatorname{trace}(\Xi(k+1)) \\
& \text { subject to } \\
& e(k+1) e^{T}(k+1) \leq \Xi(k+1)
\end{aligned}
$$

and (3), (4) and (7) (or (8)), where $e(k)=x(k)-x_{f}(k)$ is the state estimation error corresponding to time step $k$.

Remark 4: The quadratic error-constrained filtering problem defined in (10) and (11) turns out to be an optimization filter design problem, where the optimized filter parameters $L(k)$ can be calculated by minimizing the matrix sequence $\Xi(k+1)$ in the sense of matrix trace. Note that $\Xi(k+1)$ serves as the upper bound on the state estimation error matrix sequences. Another feature of such a filter design algorithm, as will be seen later, is its recursive nature that facilitates the online applications for the discrete time-varying systems with deterministic non-Gaussian noises.

For convenience of representation, we give the following notations as in [25]:

$$
\begin{aligned}
s_{1}(k):= & f(x(k))-f\left(x_{f}(k)\right)-A(k) e(k) \\
s_{2}(k-\tau):= & g(x(k-\tau))-g\left(x_{f}(k-\tau)\right) \\
& -B(k-\tau) e(k-\tau)
\end{aligned}
$$

and then the error dynamics can be obtained from (1), (2) and (9) that

$$
\begin{gathered}
e(k+1)=A(k) e(k)+B(k-\tau) e(k-\tau)+s_{1}(k)+s_{2}(k-\tau) \\
-L(k) C(k) e(k)+(D(k)-L(k) E(k)) \omega(k) .
\end{gathered}
$$

If the quadratic estimation error at time step $k$ satisfies $[x(k)-$ $\left.x_{f}(k)\right]\left[x(k)-x_{f}(k)\right]^{T} \leq \Xi(k)$, then there exists a vector $r(|r| \leq 1)$ such that

$$
x(k)=x_{f}(k)+M_{1}(k) r
$$

where $M_{1}(k)$ is a factorization of $\Xi(k)$, i.e. $\Xi(k)=M_{1}(k) M_{1}^{T}(k)$. It follows from (14) and (15) that

$$
\begin{aligned}
e(k+1)= & {[A(k)-L(k) C(k)] M_{1}(k) r+s_{1}(k) } \\
& +B(k-\tau) M_{1}(k-\tau) r+s_{2}(k-\tau) \\
& +(D(k)-L(k) E(k)) \omega(k) .
\end{aligned}
$$


In the rest of this technical note, we will develop a semi-definite programme method for the recursive filtering problem with the quadratic estimation error being enforced on the recursive process at every time step.

\section{MAIN RESUltS}

The following lemma will be used in the proofs of our main results in this technical note.

Lemma 1: Let $g_{0}(\cdot), g_{1}(\cdot), \cdots, g_{q}(\cdot)$ be quadratic functions of the variable $\zeta \in \mathbb{R}^{n}: g_{i}(\zeta) \triangleq \zeta^{T} T_{i} \zeta(i=0, \cdots, q)$, where $T_{i}=T_{i}^{T}$. If there exist $\epsilon_{1} \geq 0, \cdots, \epsilon_{q} \geq 0$ such that $T_{0}-\sum_{i=1}^{q} \epsilon_{i} T_{i} \geq 0$, then the following is true:

$$
g_{1}(\zeta) \geq 0, \cdots, g_{q}(\zeta) \geq 0 \longrightarrow g_{0}(\zeta) \geq 0 .
$$

In the following theorem, a semi-definite programme approach is developed to deal with the quadratic error-constrained filtering problem for the discrete time-varying nonlinear systems (1), (2) with bounded noises and time-delays. The nonlinearities described in Case 1 is considered in this theorem, and those in Case 2 will be discussed later. A sufficient condition is derived to guarantee the solvability of the desired filtering problem by solving a convex optimization problem.

Theorem 1: For Case 1, consider the discrete time-varying nonlinear systems (1), (2) with time-delays, bounded noises and nonlinearities described in (7). If there exist positive-definite matrix sequence $\Xi(k)>$ 0 , matrix sequence $L(k)$ and scalar sequences $\varepsilon_{i}(k)(i=1,2,3,4)$ to solve the following convex optimization problem:

$$
\begin{aligned}
& \min _{\Xi(k+1), L(k), \varepsilon_{1}(k), \varepsilon_{2}(k), \varepsilon_{3}(k), \varepsilon_{4}(k)} \operatorname{trace}(\Xi(k+1)) \\
& \text { subject to } \\
& {\left[\begin{array}{cc}
-\Xi(k+1) & \Omega(k) \\
\Omega^{T}(k) & -\Delta(k)
\end{array}\right] \leq 0}
\end{aligned}
$$

where

$$
\begin{aligned}
& \Omega(k):=\left[\begin{array}{lllll}
0 & \Omega_{1}(k) & D(k)-L(k) E(k) & I & I
\end{array}\right] \\
& \Omega_{1}(k):=(A(k)-L(k) C(k)) M_{1}(k)+B(k-\tau) M_{1}(k-\tau) \\
& \Delta(k):=\operatorname{diag}\left\{1-\varepsilon_{1}(k)-\varepsilon_{2}(k), \Delta_{1}(k), \varepsilon_{2}(k) S^{-1}(k),\right. \\
& \left.\varepsilon_{3}(k) I, \varepsilon_{4}(k) I\right\} \\
& \Delta_{1}(k):=\varepsilon_{1}(k) I-\varepsilon_{3}(k) a^{2}(k) M_{1}^{T}(k) M_{1}(k) \\
& -\varepsilon_{4}(k) b^{2}(k-\tau) M_{1}^{T}(k-\tau) M_{1}(k-\tau)
\end{aligned}
$$

then the optimization problem (10) subject to (11) and (3), (4), (7) is solvable, and the desired filter can be obtained as follows:

$$
x_{f}(k)=f\left(x_{f}(k)\right)+g\left(x_{f}(k-\tau)\right)+L(k)\left(y(k)-C(k) x_{f}(k)\right) .
$$

Proof: Defining the following augmented vector:

$$
\xi(k):=\left[1, r^{T}, \omega^{T}(k), s_{1}^{T}(k), s_{2}^{T}(k-\tau)\right]^{T}
$$

we have from (16) and (22) that $x(k+1)-x_{f}(k+1)=\Omega(k) \xi(k)$, where $\Omega(k)$ is defined in (20), and then the quadratic error constraint (11) can be rewritten as

$$
\Omega(k) \xi(k) \xi^{T}(k) \Omega^{T}(k) \leq \Xi(k+1)
$$

which, by Schur Complement, is equivalent to

$$
\begin{aligned}
& \xi^{T}(k) \Omega^{T}(k) \Xi^{-1}(k+1) \Omega(k) \xi(k)- \\
& \xi^{T}(k) \operatorname{diag}\{1,0,0,0,0\} \xi(k) \leq 0 .
\end{aligned}
$$

Similarly, $|r| \leq 1$ or $-1+r^{T} r \leq 0$, by (22), is equivalent to

$$
\xi^{T}(k) \operatorname{diag}\{-1, I, 0,0,0\} \xi(k) \leq 0 .
$$

Furthermore, it follows from (3) that:

$$
\xi^{T}(k) \operatorname{diag}\left\{-1,0, S^{-1}(k), 0,0\right\} \xi(k) \leq 0 .
$$

From (7), (12) and (13), it is easy to see

$$
\begin{aligned}
s_{1}^{T}(k) s_{1}(k) \leq & a^{2}(k) e^{T}(k) e(k) \\
= & a^{2}(k) r^{T} M_{1}^{T}(k) M_{1}(k) r, \\
s_{2}^{T}(k-\tau) s_{2}(k-\tau) \leq & b^{2}(k-\tau) e^{T}(k-\tau) e(k-\tau) \\
= & b^{2}(k-\tau) r^{T} M_{1}^{T}(k-\tau) \\
& \times M_{1}(k-\tau) r
\end{aligned}
$$

which can be rewritten by

$$
\begin{gathered}
\xi^{T}(k) \operatorname{diag}\left\{0,-a^{2}(k) M_{1}^{T}(k) M_{1}(k), 0, I, 0\right\} \xi(k) \leq 0 \\
\xi^{T}(k) \operatorname{diag}\left\{0,-b^{2}(k-\tau) M_{1}^{T}(k-\tau) M_{1}(k-\tau),\right. \\
0,0, I\} \xi(k) \leq 0 .
\end{gathered}
$$

From Lemma 1, (24) holds if there exist scalars $\varepsilon_{i}(k)(i=1,2,3$, 4) such that the following inequality is true:

$$
\begin{gathered}
\xi^{T}(k) \Omega^{T}(k) \Xi^{-1}(k+1) \Omega(k) \xi(k) \\
-\xi^{T}(k) \operatorname{diag}\{1,0,0,0,0\} \xi(k) \\
-\varepsilon_{1}(k)\left[\xi^{T}(k) \operatorname{diag}\{-1, I, 0,0,0\} \xi(k)\right] \\
-\varepsilon_{2}(k)\left[\xi^{T}(k) \operatorname{diag}\left\{-1,0, S^{-1}(k), 0,0\right\} \xi(k)\right] \\
-\varepsilon_{3}(k)\left[\xi^{T}(k) \operatorname{diag}\left\{0,-a^{2}(k) M_{1}^{T}(k) M_{1}(k), 0, I, 0\right\} \xi(k)\right] \\
-\varepsilon_{4}(k)\left[\xi ^ { T } ( k ) \operatorname { d i a g } \left\{0,-b^{2}(k-\tau) M_{1}^{T}(k-\tau) M_{1}(k-\tau),\right.\right. \\
0,0, I\} \xi(k)] \leq 0 .
\end{gathered}
$$

For $\xi(k) \neq 0$, the inequality (30) holds if and only if

$$
\begin{aligned}
\Omega^{T}(k) \Xi^{-1}(k & +1) \Omega(k) \\
+\operatorname{diag}\{ & -1+\varepsilon_{1}(k)+\varepsilon_{2}(k),-\varepsilon_{1}(k) I \\
& +\varepsilon_{3}(k) a^{2}(k) M_{1}^{T}(k) M_{1}(k) \\
& +\varepsilon_{4}(k) b^{2}(k-\tau) M_{1}^{T}(k-\tau) M_{1}(k-\tau), \\
& \left.-\varepsilon_{2}(k) S^{-1}(k),-\varepsilon_{3}(k) I,-\varepsilon_{4}(k) I\right\} \leq 0
\end{aligned}
$$

which, by Schur Complement, is equivalent to (19). The proof of this theorem is thus complete.

Next, let us consider the Case 2 where the nonlinear functions $f(\cdot)$ and $g(\cdot)$ satisfy (8). We like to derive a computationally feasible algorithm for the desired filtering problem.

Theorem 2: For Case 2, consider the discrete time-varying nonlinear systems (1), (2) with time-delays, bounded noises and nonlinearities described in (8). If there exist positive-definite matrix sequence $\Xi(k)>$ 0 , matrix sequence $L(k)$ and scalar sequences $\delta_{i}(k)(i=1,2,3,4)$ such that the following convex optimization problem is feasible:

$$
\begin{aligned}
& \min _{\Xi(k+1), L(k), \delta_{1}(k), \delta_{2}(k), \delta_{3}(k), \delta_{4}(k)} \operatorname{trace}(\Xi(k+1)) \\
& \text { subject to } \\
& {\left[\begin{array}{cc}
-\Xi(k+1) & \Omega(k) \\
\Omega^{T}(k) & -\Pi(k)
\end{array}\right] \leq 0}
\end{aligned}
$$


where

$$
\begin{aligned}
& \Pi(k):=\operatorname{diag}\left\{\Pi_{1}(k), \delta_{1}(k) I, \delta_{2}(k) S^{-1}(k), \delta_{3}(k) \mathfrak{A}^{-1}(k),\right. \\
&\left.\delta_{4}(k) \mathfrak{B}^{-1}(k-\tau)\right\} \\
& \Pi_{1}(k):=1-\delta_{1}(k)-\delta_{2}(k)-\delta_{3}(k)-\delta_{4}(k)
\end{aligned}
$$

and $\Omega(k)$ is defined in (20), then the optimization problem (10) subject to (11), (3), (4) and (8) is solvable and the desired filter in (21) can be obtained.

Proof: From (8) and (12), (13), one has

$$
\begin{aligned}
s_{1}^{T}(k) \mathfrak{A}^{-1}(k) s_{1}(k) & \leq 1, \\
s_{2}^{T}(k-\tau) \mathfrak{B}^{-1}(k-\tau) s_{2}(k-\tau) & \leq 1
\end{aligned}
$$

which can also be represented as

$$
\begin{array}{r}
\xi^{T}(k) \operatorname{diag}\left\{-1,0,0, \mathfrak{A}^{-1}(k), 0\right\} \xi(k) \leq 0 \\
\xi^{T}(k) \operatorname{diag}\left\{-1,0,0,0, \mathfrak{B}^{-1}(k-\tau)\right\} \xi(k) \leq 0 .
\end{array}
$$

Again, it follows from Lemma 1 that (24) holds if there exist scalars $\delta_{i}(k)(i=1,2,3,4)$ such that the following inequality:

$$
\begin{aligned}
& \xi^{T}(k) \Omega^{T}(k) \Xi^{-1}(k+1) \Omega(k) \xi(k) \\
& \quad-\xi^{T}(k) \operatorname{diag}\{1,0,0,0,0\} \xi(k) \\
& \quad-\delta_{1}(k)\left[\xi^{T}(k) \operatorname{diag}\{-1, I, 0,0,0\} \xi(k)\right] \\
& \quad-\delta_{2}(k)\left[\xi^{T}(k) \operatorname{diag}\left\{-1,0, S^{-1}(k), 0,0\right\} \xi(k)\right] \\
& \quad-\delta_{3}(k)\left[\xi^{T}(k) \operatorname{diag}\left\{-1,0,0, \mathfrak{A}^{-1}(k), 0\right\} \xi(k)\right] \\
& -\delta_{4}(k)\left[\xi^{T}(k) \operatorname{diag}\left\{-1,0,0,0, \mathfrak{B}^{-1}(k-\tau)\right\} \xi(k)\right] \leq 0
\end{aligned}
$$

holds. For $\xi(k) \neq 0$, the above inequality holds if and only if the inequality

$$
\begin{aligned}
& \Omega(k)^{T} \Xi^{-1}(k+1) \Omega(k) \\
& +\operatorname{diag}\left\{-1+\delta_{1}(k)+\delta_{2}(k)+\delta_{3}(k)+\delta_{4}(k),-\delta_{1}(k) I,\right. \\
& \quad-\delta_{2}(k) S^{-1}(k),-\delta_{3}(k) \mathfrak{A}^{-1}(k), \\
& \left.\quad-\delta_{4}(k) \mathfrak{B}^{-1}(k-\tau)\right\} \leq 0
\end{aligned}
$$

holds which, again from the Schur Complement, is equivalent to (33). This ends the proof.

Remark 5: In Theorem 1 and Theorem 2, the optimized filter (21) is obtained for the discrete time-varying systems with deterministic bounded noises (1), (2) at every time step $k$. Both the Lipschitz-like and ellipsoid-bounded nonlinearities are considered. Specifically, the optimization problem (10), (11) is solved by using a recursive matrix inequality approach.

As discussed in the introduction, time delays exist inherently in many practical systems which often lead to poor performance or even instability. Therefore, the time delays have been considered in (1), (2) in order to make our model more comprehensive. Nevertheless, in the case that there are no time-delays (i.e., the time-delay term $g(x(k-\tau))$ is dropped out), the corresponding results for Case 1 and Case 2 can be readily obtained, respectively, from Theorem 1 and Theorem 2, which are given in the following two corollaries without proof.

Corollary 1: For Case 1, consider the discrete nonlinear systems (1), (2) without time-delays. If there exist positive-definite matrix sequence $\Xi(k)>0$, matrix sequence $L(k)$ and scalar sequences $\varepsilon_{i}(k)(i=1$, $2,3)$ to solve the following convex optimization problem:

$$
\begin{aligned}
& \min _{\Xi(k+1), L(k), \varepsilon_{1}(k), \varepsilon_{2}(k), \varepsilon_{3}(k)} \operatorname{trace}(\Xi(k+1)) \\
& \text { subject to }
\end{aligned}
$$

$$
\left[\begin{array}{cc}
-\Xi(k+1) & \Phi(k) \\
\Phi^{T}(k) & -\Lambda(k)
\end{array}\right] \leq 0
$$

where

$$
\begin{gathered}
\Phi(k):=\quad\left[\begin{array}{cc}
0 & (A(k)-L(k) C(k)) M_{1}(k) \\
D(k)-L(k) E(k) & I
\end{array}\right] \\
\Lambda(k):=\operatorname{diag}\left\{\begin{array}{c}
1-\varepsilon_{1}(k)-\varepsilon_{2}(k), \Lambda_{1}(k), \\
\left.\varepsilon_{2}(k) S^{-1}(k), \varepsilon_{3}(k) I\right\}
\end{array}\right. \\
\Lambda_{1}(k):=\varepsilon_{1}(k) I-\varepsilon_{3}(k) a^{2}(k) M_{1}^{T}(k) M_{1}(k)
\end{gathered}
$$

then the optimization problem (10) subject to (11) and (3), (4), (7) is solved and the desired filter can be obtained as follows:

$$
x_{f}(k+1)=f\left(x_{f}(k)\right)+L(k)\left(y(k)-C(k) x_{f}(k)\right) .
$$

Corollary 2: For Case 2, consider the discrete nonlinear systems (1), (2) without time-delays. If there exist positive-definite matrix sequence $\Xi(k)>0$, matrix sequence $L(k)$ and scalar sequences $\delta_{i}(k)(i=1$, $2,3)$ such that the following convex optimization problem is feasible:

$$
\begin{aligned}
& \min _{\Xi(k+1), L(k), \delta_{1}(k), \delta_{2}(k), \delta_{3}(k)} \operatorname{trace}(\Xi(k+1)) \\
& \text { subject to } \\
& {\left[\begin{array}{cc}
-\Xi(k+1) & \Phi(k) \\
\Phi^{T}(k) & -\Upsilon(k)
\end{array}\right] \leq 0}
\end{aligned}
$$

where

$$
\begin{aligned}
\Upsilon(k) & :=\operatorname{diag}\left\{\Upsilon_{1}(k), \delta_{1}(k) I, \delta_{2}(k) S^{-1}(k), \delta_{3}(k) \mathfrak{A}^{-1}(k)\right\} \\
\Upsilon_{1}(k) & :=1-\delta_{1}(k)-\delta_{2}(k)-\delta_{3}(k)
\end{aligned}
$$

and $\Phi(k)$ is defined in (41), then the optimization problem (10) subject to (11), (3), (4) and (8) is solvable and the desired filter in (42) can be obtained.

In the following, we will show that a semi-definite programme method can be developed by using the available Matlab YALMIP toolbox and an algorithm is shown that the main results given in Theorem 1 and Theorem 2 can be converted into a computationally appealing recursive algorithm, which can help facilitating the real-time applications.

Algorithm 1: Quadratic error-constrained filter design algorithm.

Step 1: Set the initial values for the positive integer $N$, the state $\phi$, the parameters $\tau, A(k), B(k), \cdots, E(k)$ and positive-definite matrix sequence $S(k)$, positive scalars $a(k)>0$ and $b(k-\tau)>0(\mathfrak{A}(k)>0$ and $\mathfrak{B}(k)>0)$ $(k=0, \cdots, N)$. Select appropriate initial state estimate $\phi_{f}$ and quadratic error constraint matrix $\Xi_{0}$ to satisfy the conditions (3) and (4) and set $k=0$.

Step 2: Solve the convex optimization problem (18), (19) (or (32), (33)) to obtain the quadratic error constraint matrix sequence $\Xi(k+1)$, filter parameter sequence $L(k)$ and the scalars $\varepsilon_{i}(k)\left(\delta_{i}(k)\right)(i=1,2,3,4)$. From $(21)$, derive the state estimate $x_{f}(k+1)$ that satisfies the constraint inequality (11) and then set $k=k+1$.

Step 3: If $k<N$, then go to Step 2, otherwise go to Step 4.

Step 4: Stop.

Remark 6: In the Algorithm 1, a recursive filter design scheme is presented. At each step, an upper bound for the quadratic state estimation error is obtained and minimized in the sense of matrix trace. 
It is shown that the desired filter can be obtained in terms of the solutions to a discrete matrix difference inequality, which is of a form suitable for recursive computation in online applications. Note that the time-varying model addressed is comprehensive that takes into account two kinds of nonlinearities, constant time-delays as well as bounded non-Gaussian noises. Therefore, the developed algorithm can be applied to a wide range of systems. In the next section, a simulation example is presented to show the effectiveness of the proposed approach.

Remark 7: From an engineering viewpoint, the error-constrained recursive filter is efficient because only the estimated state and the output measurement from the previous time step are needed to compute the estimate for the current state. In fact, the main aim of this technical note is to develop a "non-Gaussian analogy" of the traditional Kalman filtering approach over a finite-horizon for nonlinear time-varying systems, where the quadratic error constraints in the non-Gaussian case are similar to the error-variance constraints in the Gaussian case. Based on the semi-definite programme method, an efficient algorithm is designed such that, for the admissible time-delays, nonlinear parameters and external bounded noise disturbances, the quadratic estimation error is not more than a certain optimized upper bound at every time step. On the other hand, it would be interesting to deal with the corresponding steady-state filtering problem when the system parameters become time-invariant. This is one of our future research topics.

\section{ILLUSTRATIVE EXAMPLE}

Consider the following discrete-time nonlinear systems with time delay and bounded noises:

$$
\begin{aligned}
x_{1}(k+1)= & (0.56+0.05 \sin (k)) x_{1}(k)+0.02 x_{2}(k) \\
& +0.12 \sin \left(x_{1}(k)\right)+0.1 x_{1}(k-1) \\
& +0.3 \sin (20 k) \\
x_{2}(k+1)= & 0.03 x_{1}(k)+0.54 x_{2}(k)+0.18 x_{2}(k-1) \\
& +0.1 \cos \left(x_{2}(k-1)\right)+0.28 \sin (20 k) \\
y(k)= & (1+0.05 \sin (k)) x_{1}(k)+0.45 x_{2}(k) \\
& +0.66 \sin (20 k) .
\end{aligned}
$$

Let the bounded noises $\omega(k)$ be $\sin (20 k)$ and set $S(k)=4$. Also, select the initial state and its estimate, initial quadratic error constraints as follows:

$$
\phi=\left[\begin{array}{c}
-1 \\
2
\end{array}\right], \quad \phi_{f}=\left[\begin{array}{l}
0 \\
0
\end{array}\right], \quad \Xi_{0}=\left[\begin{array}{cc}
15 & 0 \\
0 & 15
\end{array}\right] .
$$

For Case 1, we first consider the nonlinearities described in (7). Based on (46)-(48), we let $a(k)=0.2$ and $b(k)=0.12$. According to Theorem 1 and the designed algorithm, the filter parameters and state estimate at every time step $k$ can be calculated as in Table I by using the YALMIP toolbox.

Fig. 1 simulates the numerical results for the Case 1. The simulation results have illustrated our theoretical analysis.

For Case 2, we now deal with the ellipsoid nonlinearities given in (8). Assume from (46)-(48) that

$$
\mathfrak{A}(k)=\left(\begin{array}{cc}
0.2 & 0 \\
0 & 0.2
\end{array}\right), \quad \mathfrak{B}(k)=\left(\begin{array}{cc}
0.2 & 0 \\
0 & 0.2
\end{array}\right) .
$$

By using the YALMIP toolbox, it follows from Theorem 2 and Algorithm 1 that the filter parameters and state estimate at every time step $k$ can be calculated as in Table II. Similar to Case 1, the simulation results can be found in Fig. 2, which verify our desired results.

\section{CONCLUSION}

In this technical note, we have investigated the quadratic error-constrained filtering problem for discrete time-varying nonlinear systems
TABLE I

RECURSIVE RESULTS

\begin{tabular}{|c|ccc|}
\hline$k$ & $\Xi(k)$ & $L(k)$ & $x_{f}(k)$ \\
\hline 0 & {$\left[\begin{array}{cc}15 & 0 \\
0 & 15\end{array}\right]$} & {$\left[\begin{array}{l}0.4570 \\
0.4212\end{array}\right]$} & {$\left[\begin{array}{l}0 \\
0\end{array}\right]$} \\
\hline 1 & {$\left[\begin{array}{cc}13.3200 & 0 \\
0 & 13.1200\end{array}\right]$} & {$\left[\begin{array}{l}0.4546 \\
0.4242\end{array}\right]$} & {$\left[\begin{array}{l}0.1110 \\
0.1213\end{array}\right]$} \\
\hline 2 & {$\left[\begin{array}{cc}5.1554 & -4.3740 \\
-4.3740 & 13.4636\end{array}\right]$} & {$\left[\begin{array}{l}0.4546 \\
0.4242\end{array}\right]$} & {$\left[\begin{array}{l}0.4953 \\
0.5627\end{array}\right]$} \\
\hline 3 & {$\left[\begin{array}{cc}5.2457 & -4.2214 \\
-4.2214 & 12.1202\end{array}\right]$} & {$\left[\begin{array}{l}0.4545 \\
0.4243\end{array}\right]$} & {$\left[\begin{array}{l}0.5953 \\
0.6540\end{array}\right]$} \\
\hline 4 & {$\left[\begin{array}{cc}4.4778 & -3.7371 \\
-3.7371 & 12.5196\end{array}\right]$} & {$\left[\begin{array}{l}0.4545 \\
0.4243\end{array}\right]$} & {$\left[\begin{array}{l}0.3836 \\
0.4810\end{array}\right]$} \\
\hline 5 & $\vdots$ & $\vdots$ & $\vdots$ \\
\hline
\end{tabular}
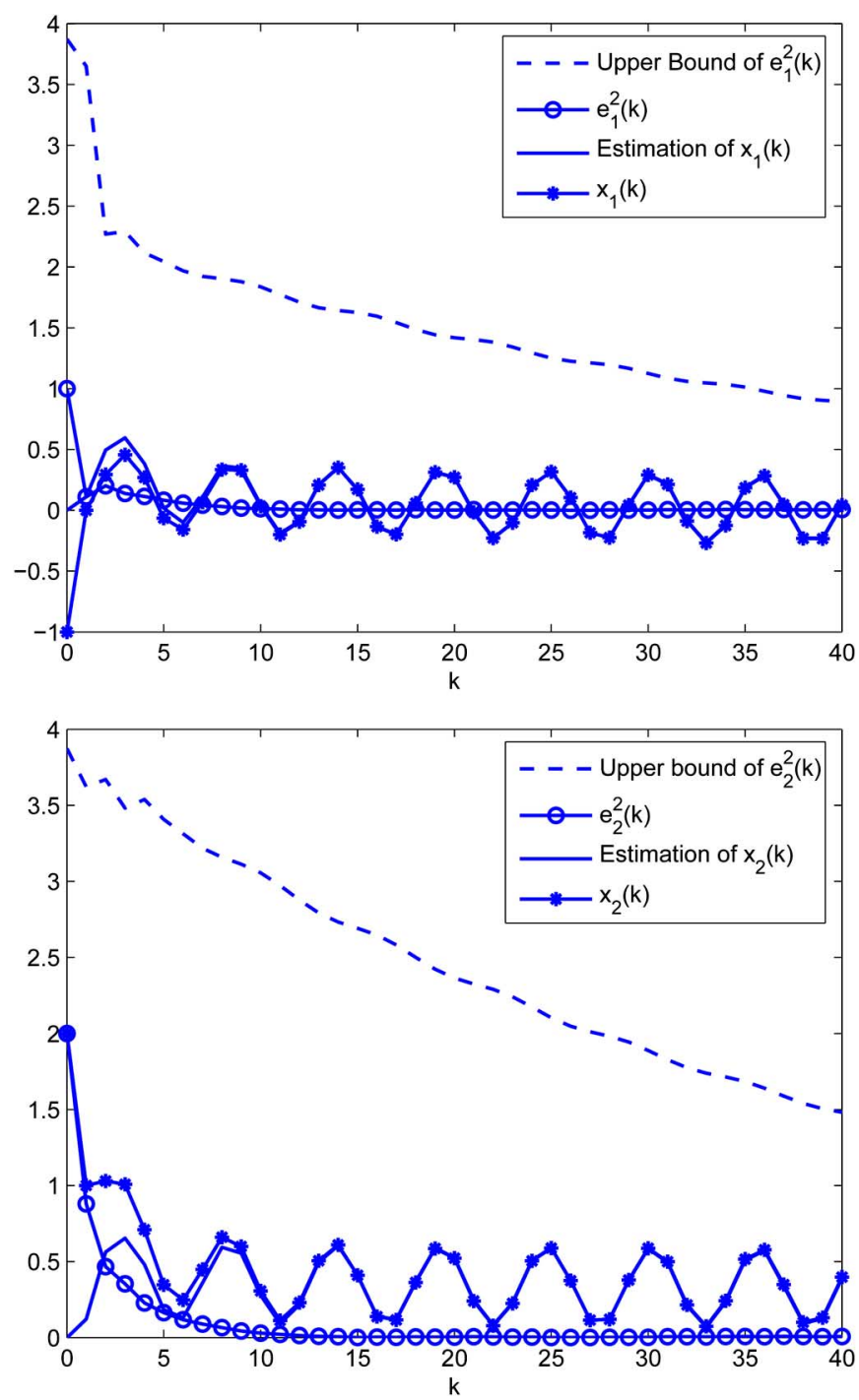

Fig. 1. Simulation results for Case 1.

with time-delays and unknown but bounded noises. The nonlinearities are described in two different ways, and the noises are constrained to reside within specified ellipsoidal sets. A recursive filter has been designed based on semi-definite programme method such that, for all admissible time delays, nonlinear parameters and external bounded noises, the quadratic error matrix is less than a minimized upper bound 
TABLE II

RECURSIVE RESULTS

\begin{tabular}{|c|ccc|}
\hline$k$ & $\Xi(k)$ & $L(k)$ & $x_{f}(k)$ \\
\hline 0 & {$\left[\begin{array}{cc}15 & 0 \\
0 & 15\end{array}\right]$} & {$\left[\begin{array}{l}0.4570 \\
0.4212\end{array}\right]$} & {$\left[\begin{array}{l}0 \\
0\end{array}\right]$} \\
\hline 1 & {$\left[\begin{array}{cc}13.3200 & 0 \\
0 & 13.1200\end{array}\right]$} & {$\left[\begin{array}{l}0.4546 \\
0.4242\end{array}\right]$} & {$\left[\begin{array}{l}0.1110 \\
0.1213\end{array}\right]$} \\
\hline 2 & {$\left[\begin{array}{cc}4.9172 & -4.3013 \\
-4.3013 & 13.0873\end{array}\right]$} & {$\left[\begin{array}{l}0.4546 \\
0.4241\end{array}\right]$} & {$\left[\begin{array}{l}0.4969 \\
0.5615\end{array}\right]$} \\
\hline 3 & {$\left[\begin{array}{cc}4.9795 & -4.0455 \\
-4.0455 & 11.5070\end{array}\right]$} & {$\left[\begin{array}{l}0.4546 \\
0.4242\end{array}\right]$} & {$\left[\begin{array}{l}0.6032 \\
0.6472\end{array}\right]$} \\
\hline 4 & {$\left[\begin{array}{cc}4.2421 & -3.5663 \\
-3.5663 & 11.9044\end{array}\right]$} & {$\left[\begin{array}{l}0.4545 \\
0.4243\end{array}\right]$} & {$\left[\begin{array}{l}0.3880 \\
0.4742\end{array}\right]$} \\
\hline 5 & $\vdots$ & $\vdots$ & $\vdots$ \\
\hline
\end{tabular}
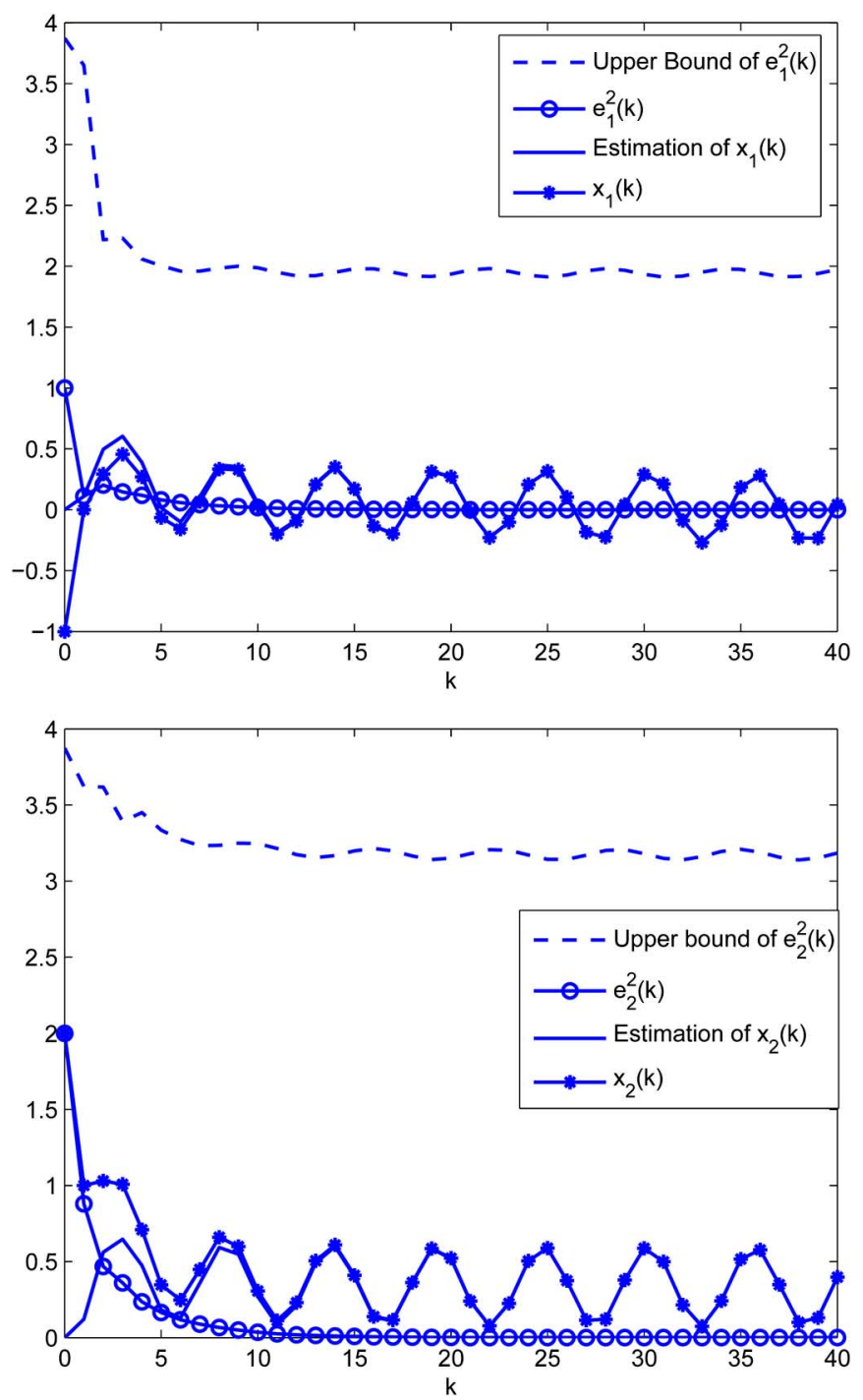

Fig. 2. Simulation results for Case 2.

in the sense of matrix trace at every time step $k$. It has been shown that the addressed filter design problem is solvable if a convex optimization problem is feasible by using the semi-definite programme method. A simulation example has been exploited in order to illustrate the effectiveness of the proposed design procedures.

\section{REFERENCES}

[1] M. V. Basin, New Trends in Optimal Filtering and Control for Polynomial and Time-Delay Systems. New York: Springer, 2008.

[2] M. V. Basin, P. Shi, D. C. Alvarez, and J. Wang, "Central suboptimal $H_{\infty}$ filter design for linear time-varying systems with state or measurement delay," Circuits, Systems and Signal Processing, vol. 28, no. 2, pp. 305-330, 2009.

[3] M. V. Basin, P. Shi, and D. Calderon Alvarez, "Central suboptimal $H_{\infty}$ filter design for linear time-varying systems with state and measurement delays," Int. J. Syst. Sci., vol. 41, no. 4, pp. 411-421, Apr. 2010.

[4] E. Fridman and U. Shaked, "On regional nonlinear $H_{\infty}$ filtering," Syst. Control Lett., vol. 29, no. 4, pp. 233-240, 1997.

[5] E. Fridman and U. Shaked, "An improved delay-dependent $\mathrm{H}$ infinity filtering," IEEE Trans. Signal Processing, vol. 52, no. 3, pp. 668-673, Mar. 2004

[6] H. Gao, J. Lam, and C. Wang, "Robust energy-to-peak filter design for stochastic time-delay systems," Syst. Control Lett., vol. 55, no. 2, pp. 101-111, 2006.

[7] H. Gao and C. Wang, "Delay-dependent robust $H_{\infty}$ and $L_{2}-L_{\infty}$ filtering for a class of uncertain nonlineaar time-delay systems," IEEE Trans. Autom. Control, vol. 48, no. 9, pp. 1661-1666, Sep. 2003.

[8] L. Ghaoui and G. Calafiore, "Robust filtering for discrete-time systems with bounded noise and parameter uncertainty," IEEE Trans. Autom. Control, vol. 46, no. 7, pp. 1084-1089, Jul. 2001.

[9] L. Guo and H. Wang, "Minimum entropy filtering for multivariate stochastic systems with non-Gaussian noises," IEEE Trans. Autom. Control, vol. 51, no. 4, pp. 695-700, Apr. 2006.

[10] T. W. Hilands and S. Thomopoulos, "Nonlinear filtering methods for Harmonic retrieval and model order selection in Gaussian and nonGaussian noise," IEEE Trans. Signal Processing, vol. 45, no. 4, pp. 982-995, Apr. 1997.

[11] Y. S. Hung and F. Yang, "Robust $H_{\infty}$ filtering with error variance constraints for discrete time-varying systems with uncertainty," Automatica, vol. 39, pp. 1185-1194, 2003.

[12] X. Lu, L. Xie, H. Zhang, and W. Wang, "Robust Kalman filtering for discrete-time systems with measurement delay," IEEE Trans. Circuits Syst. II, vol. 54, no. 6, pp. 522-526, Jun. 2007.

[13] S. Rangan and V. Goyal, "Recursive consistent estimation with bounded noise," IEEE Trans. Inform. Theory, vol. 44, no. 1, pp. 457-464, Apr. 2001.

[14] K. Reif, S. Günther, E. Yaz, and R. Unbehauen, "Stochastic stability of the discrete-time extended Kalman filter," IEEE Trans. Autom. Control, vol. 44, no. 4, pp. 714-728, Apr. 1999.

[15] K. Reif and R. Unbehauen, "The extended Kalman filter as an exponential observer for nonlinear systems," IEEE Trans. Signal Processing, vol. 47, no. 8, pp. 2324-2328, Aug. 1999.

[16] F. Schweppe, "Recursive state estimation: Unknown but bounded errors and system inputs," IEEE Trans. Autom. Control, vol. AC-13, no. 1, pp. 22-28, Feb. 1968.

[17] P. Shi, M. Mahmoud, S. K. Nguang, and A. Ismail, "Robust filtering for jumping systems with mode-dependent delays," Signal Processing, vol. 86 , no. 1 , pp. 140-152, 2006.

[18] J. C. Spall and K. D. Wall, "Asymptotic distribution theory for the Kalman filter state estimator," Commun. Stat.: Theory Methods, vol. 13, pp. 1981-2003, 1984.

[19] J. C. Spall, "Validation of state-space models from a single realization of non-Gaussian measurements," IEEE Trans. Autom. Control, vol. AC-30, no. 12, pp. 1212-1214, Dec. 1985

[20] J. C. Spall, "The Kantorovich inequality for error analysis of the Kalman filter with unknown noise distributions," Automatica, vol. 31, pp. 1513-1517, 1995.

[21] V. Suplin, E. Fridman, and U. Shaked, "Sampled-data $H_{\infty}$ control and filtering: Nonuniform uncertain sampling," Automatica, vol. 43, no. 6 , pp. 1072-1083, 2007.

[22] T.-J. Tarn and Y. Rasis, "Observers for nonlinear stochastic systems," IEEE Trans. Autom. Control, vol. AC-21, no. 4, pp. 441-448, Aug. 1976.

[23] R. Tempo, "Robust estimation and filtering in the presence of bounded noise," IEEE Trans. Autom. Control, vol. 33, no. 9, pp. 864-867, Sep. 1988.

[24] Z. Wang, D. W. C. Ho, and X. Liu, "Variance-constrained filtering for uncertain stochastic systems with missing measurements," IEEE Trans. Autom. Control, vol. 48, no. 7, pp. 1254-1258, Jul. 2003. 
[25] Z. Wang, J. Lam, and X. Liu, "Filtering for a class of nonlinear discretetime stochastic systems with state delays," J. Comput. Appl. Math., vol. 201, pp. 153-163, 2007.

[26] X. Wang and H. Poor, "Robust multiuser detection in non-Gaussian channels," IEEE Trans. Signal Processing, vol. 47, no. 2, pp. 289-30, Feb. 1999.

[27] G. Wei, Z. Wang, H. Shu, and J. Fang, "A delay-dependent approach to $H_{\infty}$ filtering for stochastic delayed jumping systems with sensor nonlinearities," Int. J. Control, vol. 80, no. 6, pp. 885-897, 2007.

[28] G. Wei, Z. Wang, H. Shu, and J. Fang, "Delay-dependent stabilization of stochastic interval delay systems with nonlinear disturbances," Syst. Control Lett., vol. 56, pp. 623-633, 2007.

[29] L. Wu, P. Shi, H. Gao, and C. Wang, " $H_{\infty}$ filtering for 2-D Markovian jump systems," Automatica, vol. 44, no. 7, pp. 1849-1858, Jul. 2008.

[30] L. Wu, J. Lam, W. Paszke, K. Galkowski, and E. Rogers, "Robust $H_{\infty}$ filtering for uncertain differential linear repetitive processes," Int. J. Adaptive Control Signal Processing, vol. 22, no. 3, pp. 243-265, 2008.

[31] L. Xie, Y. C. Soh, and C. E. de Souza, "Robust kalman filtering for uncertain discrete-time systems," IEEE Trans. Autom. Control, vol. 39, no. 6, pp. 1310-1314, Jun. 1994.

[32] L. Xie, C. de Souza, and M. Fu, " $H_{\infty}$ estimation for discrete time linear uncertain systems," Int. J. Robust Nonlin. Control, vol. 1, pp. 111-123, 1991.

[33] I. Yaesh and U. Shaked, "Min-max Kalman filtering," Syst. Control Lett., vol. 53, no. 3-4, pp. 217-228, 2004.

[34] F. Yang and Y. Hung, "Robust mixed $H_{2} / H_{\infty}$ filtering with regional pole assignment for uncertain discrete systems," IEEE Trans. Circuits Syst. I, vol. 49, no. 8, pp. 1236-1241, Aug. 2002.

[35] F. Yang, Y. Li, and X. Liu, "Robust error square constrained filter design for systems with non-Gaussian noises," IEEE Signal Processing Lett., vol. 15, pp. 930-933, 2008.

\section{D-Stability and Delay-Independent Stability of Homogeneous Cooperative Systems}

Vahid Samadi Bokharaie, Oliver Mason, and Mark Verwoerd

\begin{abstract}
We introduce a nonlinear definition of D-stability, extending the usual concept for positive linear time-invariant systems. We show that globally asymptotically stable, cooperative systems, homogeneous of any order with respect to arbitrary dilation maps are D-stable. We also prove a strong stability result for delayed cooperative homogeneous systems. Finally, we show that both of these results also hold for planar cooperative systems without the restriction of homogeneity.
\end{abstract}

Index Terms-Globally asymptotically stable (GAS), linear time-invariant (LTI).

\section{INTRODUCTION}

Due to their practical importance, Positive Systems have been the focus of a significant research effort in the Engineering, Applied Mathematics and Computational Sciences communities. The theory of pos-

Manuscript received December 02, 2009; revised May 11, 2010; accepted August 20, 2010. Date of publication September 13, 2010; date of current version December 02, 2010. This work was supported by Science Foundation Ireland (SFI) Grant 08/RFP/ENE1417 and by the Irish Higher Education Authority (HEA) PRTLI Network Mathematics Grant. Recommended by Associate Editor L. Marconi.

V.S. Bokharaie is with the Hamilton Institute, National University of Ireland, Maynooth, Ireland (e-mail: vahid.bokharaie@nuim.ie).

O. Mason and M. Verwoerd are with the Hamilton Institute, National University of Ireland, Maynooth, Ireland (e-mail: oliver.mason@nuim.ie; mark.verwoerd@nuim.ie).

Digital Object Identifier 10.1109/TAC.2010.2076334 itive linear time-invariant (LTI) systems is now well understood; however, for many applications of positive systems, factors such as nonlinearities, uncertainties and delays need to be taken into account. The work of this note is concerned with extending aspects of the stability theory of positive LTI systems to classes of nonlinear and delayed systems. Specifically, we shall show that two key stability properties of positive LTI systems extend directly to cooperative systems defined by vector fields that are homogeneous with respect to an arbitrary dilation map.

The LTI system $\dot{x}(t)=A x(t)$ is positive if and only if the matrix $A$ is Metzler, meaning that all of its off-diagonal elements are nonnegative. It is well known [7] that a positive LTI system is globally asymptotically stable (GAS) if and only if $\dot{x}(t)=D A x(t)$ is asymptotically stable for any diagonal matrix $D$ with positive diagonal entries. This latter property is usually referred to as D-stability.

For positive time-delayed systems, it was shown in [4] that the delayed positive linear system $\dot{x}(t)=A x(t)+B x(t-\tau)$, where $A$ is Metzler and $B$ is nonnegative, is GAS for all values of the delay $\tau \geq 0$ provided the system with zero delay $\dot{x}(t)=(A+B) x(t)$ is GAS. In this regard, interesting results providing similar stability conditions for classes of positive systems defined by functional and integrodifferential equations have recently appeared in [8], [9].

Recently, it was shown in [2] that the results for positive LTI systems mentioned in the previous paragraph also hold for cooperative systems that are homogeneous of degree zero with respect to the standard dilation map on $\mathbb{R}^{n}$. The principal contribution of the current note is to further extend these results to cooperative systems that are homogeneous of any degree with respect to an arbitrary dilation map. It should be noted that the definition of D-stability considered here is considerably more general than that investigated in [2]. In particular, this allows the results of the current paper to be applied to cooperative systems that are not necessarily homogeneous. In the same vein, we show that the assumption of homogeneity is not necessary for planar cooperative systems. Removing this assumption for higher dimensional systems is the subject of ongoing work by the authors.

The layout of the note is as follows. In Section II we introduce notation, standard definitions and the key results needed for our later analysis. In Section III we introduce a nonlinear extension of the concept of D-stability and demonstrate that GAS homogeneous cooperative systems have this property. A strong stability result for delayed systems is then given in Section IV. In Section V we show that the homogeneity assumption is not required for planar systems and finally, in Section VI we present our conclusions.

\section{MATHEMATICAL BACKGROUND}

Throughout the paper, $\mathbb{R}$ and $\mathbb{R}^{n}$ denote the field of real numbers and the vector space of all $n$-tuples of real numbers, respectively. $\mathbb{R}^{n \times n}$ denotes the space of $n \times n$ matrices with real entries. For $x \in \mathbb{R}^{n}$ and $i=1, \ldots, n, x_{i}$ denotes the $i^{t h}$ coordinate of $x$. Similarly, for $A \in \mathbb{R}^{n \times n}, a_{i j}$ denotes the $(i, j)^{t h}$ entry of $A$. Also, for $x \in \mathbb{R}^{n}$, $\operatorname{diag}(x)$ is the $n \times n$ diagonal matrix in which $d_{i i}=x_{i}$.

Throughout the paper, we shall be concerned with positive systems and with the stability properties of the equilibrium at the origin. For this reason, when we say that a system is Globally Asymptotically Stable, GAS for short, we mean that the origin is a GAS equilibrium of the system with respect to initial conditions in the non-negative orthant $\mathbb{R}_{+}^{n}:=\left\{x \in \mathbb{R}^{n}: x_{i} \geq 0,1 \leq i \leq n\right\}$.

The interior of $\mathbb{R}_{+}^{n}$ is denoted by $\operatorname{int}\left(\mathbb{R}_{+}^{n}\right)$ and its boundary by $b d\left(\mathbb{R}_{+}^{n}\right):=\mathbb{R}_{+}^{n} \backslash$ int $\left(\mathbb{R}_{+}^{n}\right)$. For vectors $x, y \in \mathbb{R}^{n}$, we write: $x \geq y$ if $x_{i} \geq y_{i}$ for $1 \leq i \leq n ; x>y$ if $x \geq y$ and $x \neq y ; x \gg y$ if $x_{i}>y_{i}, 1 \leq i \leq n$. 